\title{
A Novel Methodology For In-Process Monitoring Of Flow Forming
}

\author{
Andrew Appleby ${ }^{1,2, a)}$, Alastair Conway ${ }^{2}$ and William Ion ${ }^{1}$ \\ ${ }^{1}$ Department of Design, Manufacture E Engineering Management, University of Strathclyde, James Weir Building, \\ 75 Montrose Street, Glasgow, G1 1XJ \\ ${ }^{2}$ Advanced Forming Research Centre, University of Strathclyde, 85 Inchinnan Drive, Inchinnan

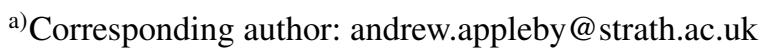

\begin{abstract}
Flow forming (FF) is an incremental cold working process with near-net-shape forming capability. Failures by fracture due to high deformation can be unexpected and sometimes catastrophic, causing tool damage. If process failures can be identified in real time, an automatic cut-out could prevent costly tool damage. Sound and vibration monitoring is well established and commercially viable in the machining sector to detect current and incipient process failures, but not for FF. A broad-frequency microphone was used to record the sound signature of the manufacturing cycle for a series of FF parts. Parts were flow formed using single and multiple passes, and flaws were introduced into some of the parts to simulate the presence of spontaneously initiated cracks. The results show that this methodology is capable of identifying both introduced defects and spontaneous failures during flow forming. Further investigation is needed to categorise and identify different modes of failure and identify further potential applications in rotary forming.
\end{abstract}

\section{INTRODUCTION}

This work constitutes the first phase of a study into acoustic monitoring of rotary forming. It is an investigation of the feasibility of using acoustic sensing for in-process monitoring of flow forming (FF). A stand-off microphone was used to record the sound signal of FF parts with defects.

\section{Flow forming}

FF is an incremental rotary forming (IRF) process that forms rotationally symmetrical parts, such as driveshafts, pressure cylinders and helicopter rotors. IRF describes a family of technologies that create near net-shape parts by gradual cold-working in series of cyclical deformations. Each pass of the tool causes a small, precise deformation. The accumulated passes can reach high levels of deformation to produce complex parts with high precision.

The workpiece is a hollow tube which is mounted on a cylindrical mandrel with one end fixed. The mandrel rotates the workpiece, which is then deformed by three rollers which are staggered sequentially in the radial and axial dimensions. Each area of material is contacted by the rollers one after another, with each subsequent roller closer to the mandrel. The mandrel provides all of the energy in the system; the rollers are free-spinning.

Flow forming is non-deterministic and hard to model. The deformation is complex and three dimensional failures occur when the deformation exceeds the material formability. These manifest as different kinds of fractures, which can be unexpected and catastrophic, presenting a potential risk of damage to tooling and machine downtime.

Current practice uses a trial-and-error approach to optimise machine parameters when working near the forming limit. There is no existing method to predict, detect or categorise failures in-process. There is an opportunity for improved monitoring of flow forming to advance understanding of the process and improve its controllability and commercial viability. A live-monitoring and detection system that could detect defects occurring and categorise them by size and location would be beneficial for improved understanding of the process behaviour and ultimately for commercial applications of the technology. 


\section{Process Monitoring}

On-line process monitoring is a well-established field that has been applied to a wide variety of processes. The aim usually is to extract meaningful data from some aspect of the process and use this to detect and predict process issues. There are a variety of relevant existing monitoring technologies, used on a range of processes. The monitored variables can be force, torque, sound, vibration, acoustic emission, temperature, vibration, etc.

Acoustic emission (AE), sound and vibration are often suitable for machining monitoring, where researchers have attempted to characterise the condition of the tooling, the behaviour of the process, and the quality of the output. [1] These technologies are generally simple to install on existing equipment and are highly effective, which has contributed to making them well-established and commercially viable in a variety of industrial applications to detect current and incipient process failures.

$\mathrm{AE}$ has been used in various areas of machining monitoring, for example to identify grease contamination in lathe bearings[2] and for face milling and chemical-mechanical planarisation[3]. In addition, AE has been widely used for machine tool condition monitoring (TCM). $[4,5]$

Monitoring of sheet metal forming has shown that AE sensing can characterise plastic deformation in some circumstances. [6] described how the complex movement and interaction of dislocations produces high frequency emissions, easily detectable during high deformation levels or uniaxial yield. Notably, it was found to be hard to detect the AE from smaller deformations because only a small proportion of the AE can be captured. Detection of failures in sheet metal forming has also been shown - cracking, galling, stick slip friction conditions, and tool wear were all detected by AE.[7, 8]

Monitoring of bulk metal forming has been an area of limited research. Behrens and Just [9] state that passive ultrasonic testing is effective for cold forging because of the AE caused spontaneously by material flow can be used to identify fracture. The sources of these emissions are described as intra-crystal friction, dynamic recrystallisation and plastic deformation, although these were difficult to distinguish as was also found by Hao, Ramalingam, and Klamecki [6]. Difficulties in the distinction of distinguishing valuable information from background noise were reportedly overcome by statistical processing. Schneider et al. [10] also made a similar experiment, using AE to identify the forming limits in upsetting of high-alloy steels with the motivation of reducing surface defects in open-die forging. Similar results have been obtained for hot and cold upsetting in Mg-Al alloys and steels. [11, 12].

Monitoring of sheet and bulk metal forming generally consists of a series of near-identical operations (stamping, punching forging, etc.). This makes it logical to collect a signal for each operation and then analyse the signal for drifting trends over time or sudden anomalies.

Note that for most machining and TCM applications, there are essentially two parts (tool and workpiece) of which one is rotating and one is fixed. For sheet and bulk metal forming, there are small numbers of moving parts, which generally have large areas of contact, giving a good acoustic pathway for monitoring sounds. These set-ups make it practical to attach a contact sensor (such as for AE or vibration sensing) to the fixed part - the sensor is near the site of activity with an uninterrupted acoustic pathway. For FF, it is impossible to access the site of deformation in this way due to the rotation of both tool and workpiece. Furthermore, the area of contact between the tool and workpiece is constantly moving and can change shape during the process.

Monitoring of rotating machinery poses significant challenges: The site of interest is often inaccessible to fixed or contact sensors because of the moving parts, and there are typically high levels of background noise. While vibration is considered by Wymore et al. [13] to be inferior to $\mathrm{AE}$ in terms of sensitivity, it does not need to be placed so close to the source to be effective. Vibration monitoring has been widely used for monitoring of rotating machinery, for example wind turbine gearboxes. Contact accelerometers are typically mounted on the gearbox housing and can therefore suffer poor signal-to-noise ratio, but are effective for fault detection and prognosis. [14]

$\mathrm{AE}$ and vibration are usually recorded with accelerometers, which measure vibrations through physical contact. They need to be attached to the vibrating part. For IRF, the sound reaching a contact accelerometer would need to propagate from the site of deformation through a series of interfaces (e.g. through the roller and bearings to the housing) resulting in a weak, noisy signal. Microphones are a standoff sensor which only need to be located close to the source of sound. This makes them inexpensive and simple to install without interfering with the process.

The use of stand-off microphones has been demonstrated for TCM in conjunction with other signals $[15,16]$ and alone. $[17,18]$ The omnidirectional nature of microphones gives the capacity to collect a large quantity of data: if different elements of the process produce different frequencies, sound data can capture an overview of the entire process. This can lead to background noise issues because of their unselective nature, meaning post-processing is important.[15] 
The recording of audio signals is therefore useful as an approach which is simple to install without interference in the machine and has a track record of providing useful process information. The omnidirectional nature means that it captures the noise of the whole process - this is beneficial in a poorly understood process where it is not known which elements of the process will relate to failure.

\section{Scope and Aims}

IRF processes need better instrumentation to improve control and reliability. Many process monitoring technologies are available, but most are impractical for IRF. Multiple rotating elements mean that contact sensors are difficult to apply, this rules out the detection of low amplitude AE from microstructural deformations. Acoustic monitoring using a microphone offers an approach for collecting data from the whole process. This will aim to capture the noise of macro-scale deformation and fracture as it propagates directly from the site to the air.

The scope of this paper is the investigation of acoustic sensing for detecting failure in FF. This work aims to determine if acoustic monitoring can collect any useful signal from the FF process, what the key frequency region is, and whether fracture is detectable.

\section{METHODOLOGY}

The key input variables are the deformation rate and the presence of defects. Forming under a range of radial deformation rates will alter the likelihood of failure and the failure type. Using artificial defects will allow a controlled investigation of the acoustic outputs of fracture. The outcome is the quality of the formed parts.

\section{Equipment Selection and Set-up}

The sound of major, catastrophic fractures is clearly audible to the machine operator from outside the machine housing during forming. Smaller fractures can be sometimes be heard, but it is difficult to distinguish between noise from the machine and ambient noises, e.g. from other machines in the same environment. Human acoustic range is in the region of $20 \mathrm{~Hz}$ to $20 \mathrm{kHz}$ : an acoustic monitoring system with a good response across the same frequency should therefore be able to capture the same information as an operator and explore outside of this range.

(a)

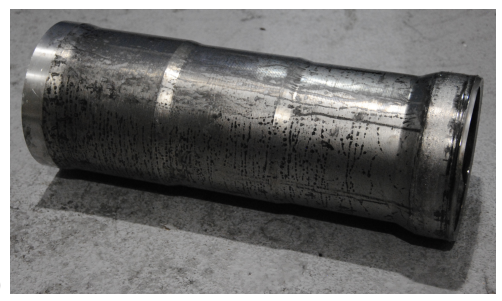

(b)

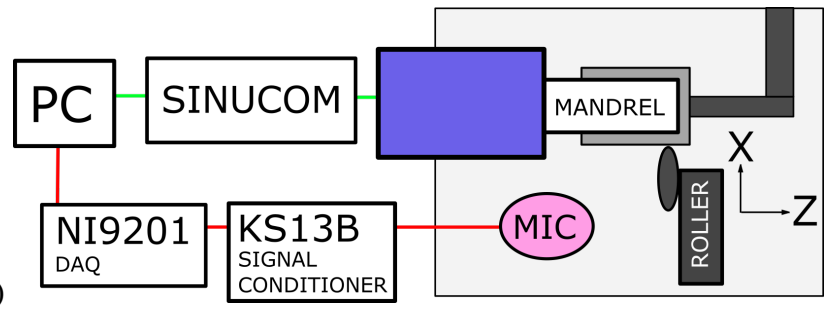

FIGURE 1. (a) Three-stage reduction formed part. (b) Equipment set-up.

Figure 1 shows the configuration of equipment used. The PCB Piezotronics 130E21 microphone was driven with a Kistler 5134B signal conditioner. The data was recorded using a National Instruments 9201 DAQ interfaced with National Instruments LabView SignalExpress. This is an inexpensive, portable system capable of capturing the same acoustic information as an operator. The machine positions and forces were recorded with the Siemens Sinumerik system, using the Sinucom software to record the data.

Data processing was done in Matlab 2016b. Initial investigations showed that the sound signal of frequencies above $380 \mathrm{~Hz}$ was dominated by the spindle noise, machine noise of interest was in the range below this. The initial data (recorded at $50 \mathrm{kHz}$ ) was therefore low-pass filtered to avoid aliasing and downsampled to $5 \mathrm{kHz}$. The data was assessed in the time and frequency domains using discrete Fourier transorms to produce spectrograms. Time-matching of the data was achieved using the Sinucom recordings of roller positions through the process.

The underlying data can be accessed at: http://dx.doi.org/10.15129/e1236424-3131-471f-a13b-25980424ca8d 


\section{Part Selection and Fault Introduction}

FF has a nominal operating range of between $20 \%$ and $80 \%$ deformation. Forming near or past the upper limit tends to cause failure. The FF program was the same for all of the trials, a three-stage reduction in annealed 6061 Aluminium as shown in Figure 1 (a). The three lands are reductions in thickness of $25 \%, 50 \%$ and $75 \%$. This allows the investigation of a range of deformation rates. The relative softness of the material limited the risk of tool damage and spontaneous fracturing.

For some of the trials, the same forming program was repeated in a second pass with a further $0.5 \mathrm{~mm}$ reduction in the thickness of each land. This produced marginal forming rates of $7 \%, 10 \%$ and $21 \%$, making the total deformation $31 \%, 55 \%$ and $80 \%$. Operating under sustained high deformation allows the investigation of spontaneous fracturing.

The sound from the deformation under normal conditions is not audible, therefore defects were introduced to investigate the audio response to failure. If an audio response to failure is consistently detectable, it would allow a sound-based emergency stop mechanism to protect the tooling.

\section{RESULTS}

\section{Characterising the audio signal}

The sound signature of each flow formed part shares the same general characteristics. Figure 2(a) shows the sound recording during flow forming alongside the machine's position recordings. When the machine starts, a large amount of noise is generated by the motor spindle, bearing and coolant system. This is visible as a band of frequencies from $380 \mathrm{~Hz}$ upwards. Large movements of the rollers in the radial direction produce noise at $190 \mathrm{~Hz}$ at the beginning and end of flow forming.

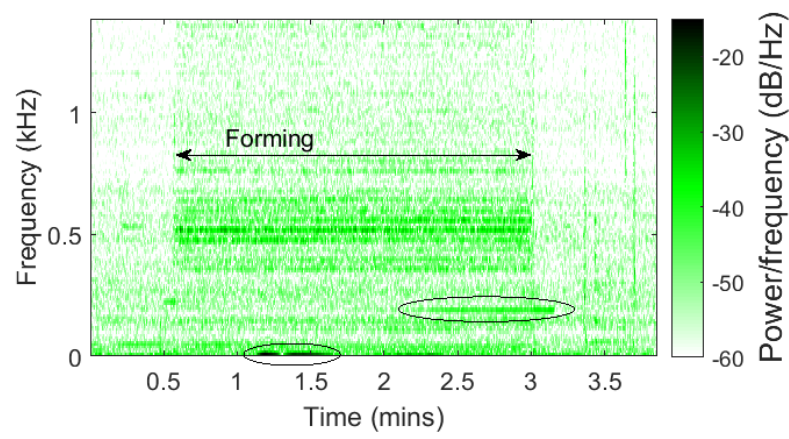

FIGURE 2. The spectrogram of a single pass FF. The band of high intensity frequencies comes from the spindle motor of the machine. The circled areas show low frequency rumbling and the sound from the rollers' hydraulic slides.

Other obvious features of the signal are: Intermittent signal across all trials at $50 \mathrm{~Hz}$ - this is likely to be electrical interference. Low frequency (sub $5 \mathrm{~Hz}$ ) rumbling occurs in all trials - note that the spindle speed is $5 \mathrm{~Hz}$. The spectrogram approach allows the identification of frequencies associated with damage or failure. In the parts with defects, there were a number of relevant frequencies. The regions of 10, 20, 35, 113, 118, 275 and $313 \mathrm{~Hz}$ show significant responses. The response in these frequency areas is related to process failure and high deformation.

\section{Signal of Process Failures - Single Pass}

A part was formed with defects added to the preform. Two holes were drilled, of $2 \mathrm{~mm}$ and 4 mm diameters, offset $100 \mathrm{~mm}$ and $200 \mathrm{~mm}$ respectively from the capped end. The part formed successfully - the material flowed over the holes, leaving them invisible externally but unchanged on the interior face. The holes did not propagate into larger fractures due to the high formability of the material and the small size of the defects. Figure 3(a) shows the spectrogram of the forming, with disturbances at $50 \mathrm{~s}$ and $1 \mathrm{~m} 44 \mathrm{~s}$ into the process. Comparison with the roller positions shows that the noise at $1 \mathrm{~m} 44 \mathrm{~s}$ matches the time when the roller passes over the second defect. But the elevated noise at $50 \mathrm{~s}$ does not align with the roller passing over the first hole, which appears to make no noise. Therefore this noise is likely caused by the increase in deformation from 25 to $50 \%$. 
(a)

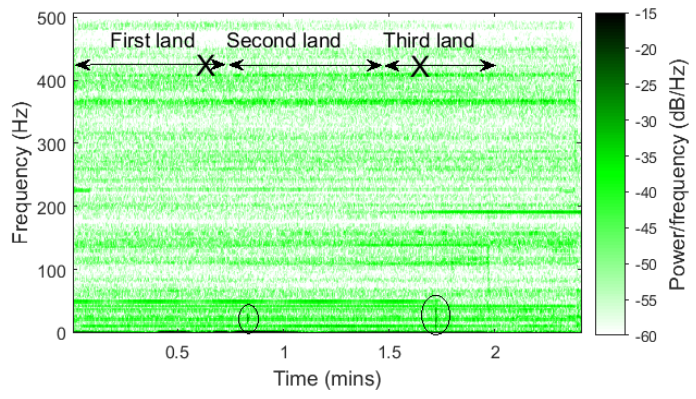

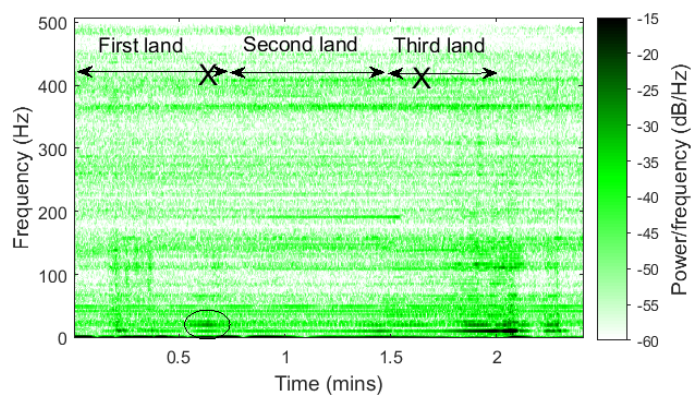

(b)

FIGURE 3. (a) Single pass FF with holes drilled in preform. The rollers pass the holes at $38 \mathrm{~s}$ and $1 \mathrm{~m} 44 \mathrm{~s}$. Note disturbances at $50 \mathrm{~s}$ and $1 \mathrm{~m} 44 \mathrm{~s}$. (b) Single pass FF with slots cut in preform. Note disturbance at $38 \mathrm{~s}$.

A second part was formed with larger defects. Two slots were cut in the material with a bench saw at 100 and $200 \mathrm{~mm}$ from the capped end. The first was $7 \mathrm{~cm}$ long and $8 \mathrm{~mm}$ deep at its deepest, the second $9 \mathrm{~cm}$ long and through the preform at its deepest point. When passing the first slot, the material formed over the defect without failing (see Figure 5 (a)). The thickness of the formed part at this point was $7.1 \mathrm{~mm}$. The material deformed over the slot, almost sealing it over. At the second slot the part thickness was $2.4 \mathrm{~mm}$ : the crack propagated around the circumference (see Figure 5 (b)). The machine was emergency stopped.

Figure 3(b) shows the presence of significant disturbances in the audio signal. The noise starts before the roller crosses the defect. As the part is deformed, the response is affected by the large defect ahead (in the $\mathrm{z}$ dimension) of the roller. Therefore the part begins to distort before the roller reaches the defect. It can be seen that the presence of such significant defects can be readily detected in the audio signal.

\section{Signal of Process Failures - Second Pass}

Figure 4(a) shows a second pass flow forming with no added defects. The part formed successfully without fracturing despite the high levels of deformation. In the third land, under very high deformation (80\%) the surface of the part begins to roughen and break down (see Figure 5(c)).

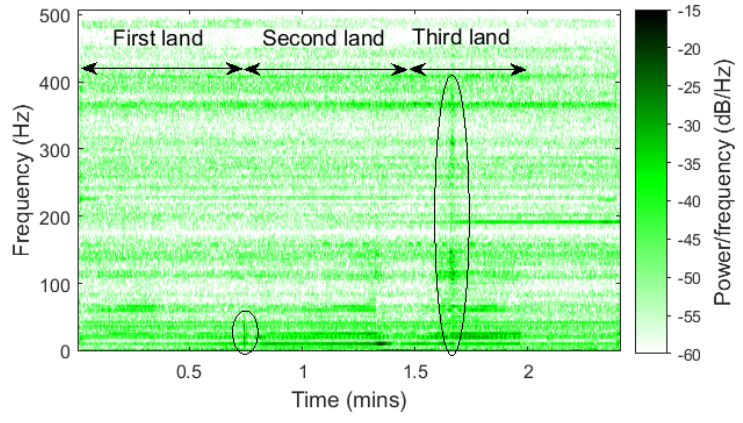

(a)

FIGURE 4. Second pass formings: (a) With no defects. Note disturbances (circled) at $45 \mathrm{~s}$ and $1 \mathrm{~m} 40 \mathrm{~s}$. (b) With drilled holes (marked by Xs). Disturbances at $24 \mathrm{~s}, 1 \mathrm{~m} 10 \mathrm{~s}$ and $1 \mathrm{~m} 45 \mathrm{~s}$ (circled).

Figure 4(a) The noise at $45 \mathrm{~s}$ is at the same time as the increasing deformation at the transition from the first to second land. The noise at $1 \mathrm{~m} 40 \mathrm{~s}$ occurs when the roller passes over the area of surface breakdown. This suggests that the noise is related to the instigation of failure.

Another part was formed, identical but with a $4 \mathrm{~mm}$ hole drilled at the mid point of each land. When the roller passes over the first two holes, the signal shows significant disturbance in the 100-500 Hz region. Figure 4(b) shows that there is a strong response at $113 \mathrm{~Hz}$ at each of the holes at $24 \mathrm{~s}$ and $1 \mathrm{~m} 10 \mathrm{~s}$. The part breaks in the third land at $1 \mathrm{~m} 45 \mathrm{~s}$; the fracture occurred at a similar position to the surface roughening detected in Figure 4(a). 
(a)

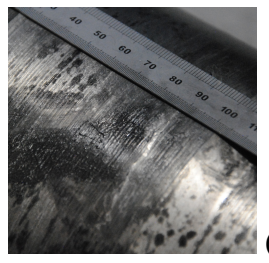

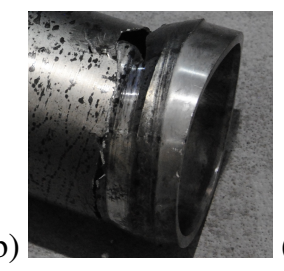

(c)

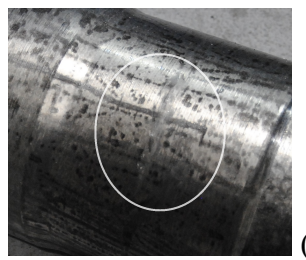

(d)

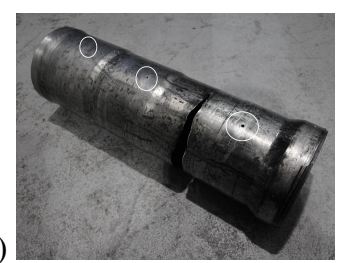

FIGURE 5. Defects in formed parts: (a) A slot cut which was covered over in forming, (b) Circumferential crack started by a slot cut, (c) Surface breakdown, a prelude to spontaneous fracture, (d) A second pass FF with drilled holes (circled) and spontaneous fracture.

\section{CONCLUSIONS}

Several conclusions can be drawn: Firstly, it was demonstrated that the equipment and method can gather useful information about the forming process. The key region for investigation is sub $400 \mathrm{~Hz}$ Both small and large defects cause disturbances in the audio signal. Spontaneous surface breakdown under high deformation (a precursor to failure) also had an impact in the audio signal. This paves the way for an acoustic system which could potentially detect fractures and near failures. This would enable a better real-time understanding of the FF process and could prevent catastrophic failures and the associated tooling damage.

\section{FURTHER WORK}

This is a promising approach for monitoring of a process that has no other developed options for monitoring. However it is important to note that not all defects cause noises and not all noises indicate defects. Questions remain, most importantly: how does the audio response relate to the defect size, wall thickness, deformation rate and part type? The next stage of development is to use this monitoring method to identify and categorise specific failure modes. A series of experiments is planned to identify and categorise failures based on their audio signals. These will use a planned approach to optimise the methodology and aim to establish a signal processing approach that allows consistent identification of fracture events.

\section{REFERENCES}

[1] R. Teti, K. Jemielniak, G. O’Donnell, and D. Dornfeld, CIRP Ann. - Manuf. Techn. 59, 717-739 (2010).

[2] S. Saravanan, G. S. Yadava, and P. Rao, Int J Adv Manuf Technol (2006).

[3] D. E. Lee, I. Hwang, C. M. O. Valente, J. F. G. Oliveira, and D. A. Dornfeld, Int. J. Mach. Tool Manu. 46, 176-188 (2006).

[4] Q. Ren, M. Balazinski, L. Baron, K. Jemielniak, R. Botez, and S. Achiche, Inform. Sciences 255, 121-134 (2014).

[5] P. S. Pai and P. K. R. Rao, Int. J. Prod. Res. 40, 1081-1093 (2002).

[6] S. Hao, S. Ramalingam, and B. E. Klamecki, J. Mater. Process. Technol. 101, 124-136 (2000).

[7] T. Skåre and F. Krantz, Wear 255, 1471-1479 (2003).

[8] T. Jayakumar, C. K. Mukhopadhyay, S. Venugopal, S. L. Mannan, and B. Raj, Journal of Materials Processing Technology 159, 48-61 (2005).

[9] B.-A. Behrens and H. Just, Journal of Materials Processing Technology 125-126, 295-301 (2002).

[10] V. Schneider, G. Hirt, a. Brethfeld, G. Lehmann, and U. Diener, Materialwissenschaft und Werkstofftechnik 38, 274-287 (2007).

[11] I. El-Galy, I. Pfeiffer, and B.-A. Behrens, Materials Science and Technology Conference and Exhibition 2009 4, 2224-2235 (2009).

[12] B.-A. Behrens, a. Santangelo, and C. Buse, Production Engineering 7, 423-432 (2013).

[13] M. L. Wymore, J. E. V. Dam, H. Ceylan, and D. Qiao, Renew. Sustainable Energy Rev. 52, 976-990 (2015).

[14] M. Nie and L. Wang, Procedia CIRP 11, 287-290 (2013).

[15] D. R. Salgado and F. J. Alonso, Int. J. Mach. Tool Manu. 47, 2140-2152 (2007).

[16] E. Kuljanic, G. Totis, and M. Â. Sortino, 23, 1704-1718 (2009).

[17] N. Seemuang, T. Mcleay, and T. Slatter, Int. J. Adv. Manuf. Tech. (2016), 10.1007/s00170-015-8303-8.

[18] W. L. Weingaertner, R. B. Schroeter, M. L. Polli, and J. d. O. Gomes, J. Mater. Process. Technol. (2006). 